Research Article

\title{
Improved Point Dipole Model for Subwavelength Resolution Scattering Near-Field Optical Microscopy (SNOM)
}

\author{
Guizhen Lu $\mathbb{D}^{1},{ }^{1}$ Ruiqi Zhao, ${ }^{1}$ Hongcheng Yin $\mathbb{D}^{2},{ }^{2}$ Zhihe Xiao, ${ }^{2}$ and Jing Zhang ${ }^{2}$ \\ ${ }^{1}$ College of Electronic Engineering, Communication University of China, Beijing 100024, China \\ ${ }^{2}$ Science and Technology on Electromagnetic Scattering Laboratory, Beijing 100039, China \\ Correspondence should be addressed to Guizhen Lu; luguizhen@cuc.edu.cn
}

Received 23 October 2019; Revised 6 January 2020; Accepted 27 January 2020; Published 30 March 2020

Academic Editor: Renato Cicchetti

Copyright (c) 2020 Guizhen Lu et al. This is an open access article distributed under the Creative Commons Attribution License, which permits unrestricted use, distribution, and reproduction in any medium, provided the original work is properly cited.

High-resolution microscopy technique is of significant importance for studying nanomaterials. It is necessary to understand the near-field interaction between the probe and substrate materials in order to get the fine structure of the nanomaterial in the subwavelength scale. The numerical methods such as FDTD, FEM, and MoM are inefficient for the SNOM problems because of the illness of the impedance matrix. The analytic method can only be used for some simple objects such as sphere. Here, a quasianalytical method is developed, in which the analytic formula is refined to adapt to various shapes of the probe approaching the curve of SNOM. By this way, it is helpful in comparing the performance of different probes and giving us a direction to design a new type probe in SNOM. As an application, the developed method is used to study the contrast in the SNOM for the interface between the two different surfaces that have different materials and topography.

\section{Introduction}

Scanning near-field optical microscopy (SNOM) is a developed subwavelength scanning imaging technique that can be used to study the subwavelength structure. By the nearfield mechanism, the high-resolution microscopy can be used, which overcomes the conventional spatial resolution constraints due to the far-field method [1].

Apertureless scanning near-field optical microscopy is obtained by using near-field scattering, and the scattered field is related with the near-field interaction between the probe and substrate materials. The spatial resolution relies on the sharpness of the probe tip and is independent of the incidence field wavelength. In order to explain the SNOM imaging, it is necessary to study the near-field interaction of the probe tip and sample surface. The understanding of the SNOM imaging can provide us useful information about materials and structure of samples [2].

Recently, the high spatial resolution of SNOM, which is independent of wavelength, has been implemented in various practical applications, such as nanoscale vibration spectroscopy [3], identification of the material type according to the SNOM image on the edge of the sample [4], nanoimaging of plasma polaritons in nanostructures, such as graphene-layered structure [5-9] and nanoantennas [10, 11], contrast and imaging performance in photo-induced force microscopy [12], quantitative measurement of nano-optical properties $[13,14]$, sensitivity of SNOM nanometer imaging with temperature [15], and tomographic NF imaging [16-18].

In the development of the SNOM theory [11, 19-33], the point dipole model (PDM) is presented to describe the nearfield interaction. The PDM is a simple analytic formula and can explain many phenomena experimentally observed, such as material contrast, phonon-polarization resonance, and blue shift with increasing probe-sample distance [34]. However, for the SNOM probe in experiment, the PDM cannot give a good description to the near-field contrasts. Specially, the near-field approach curves have discrepancy compared with experiment [34-36].

The computational electromagnetic methods have advantages in adapting the geometry of the studied problems. However, it is time-consuming for the near-field interaction between the probe tip and the sample. 
Because the near-field scattering is very weak and the vibration of the near-field probe tip is required in order to extract the higher harmonic signal demodulation, numerical computing is seldom considered [4, 37].

In this paper, an improved PDM (IPDM) is proposed, in which the IPDM has the similar formula as PDM except that the power index of sphere radius is different from the conventional PDM formula [34, 37, 38]. The incident and scattering fields of the near-field probe on the surface of the plane sample after incident by plane wave are shown in Figure 1. In Section 2, first, we discussed the polarizability of metal sphere and dielectric sphere and discussed the equivalent between metal sphere radius and permittivity of the dielectric sphere in free space in Section 2.1. Next, considering the SNOM case, the relationship between the metal sphere on the plane sample and the dielectric sphere on the plane sample is discussed in Section 2.2, in which the relation of the equivalent metal radius and permittivity of dielectric sphere is given. Third, in order to extend the relationship to any shape near the probe, we propose the IPDM method in Section 2.3, in which the polarizability of probe can be obtained by the computational method and using the numerical results, the parameters in IPDM can be obtained with the optical fitting method. The validations of the IPDM are performed for the spheroid and conical probes. Finally, by using the IPDM method, the contrasts of SNOM are studied and the resolution of SNOM can be described by the equivalent radius of the metal sphere.

\section{PDM Method for the Sphere}

The PDM uses a dielectric sphere instead of the SNOM probe to analyze the interaction between the SNOM probe and sample structure [39]. This model has the advantages of simplicity and clear physical concept. However, due to the simplification of the model's geometric structure, there is a certain deviation from the actual measurement data of the probe. In view of the shortcomings of the PDM, a finite dipole model (FDM) is proposed, which uses ellipsoid instead of sphere, and thus is closer to the actual probe in terms of geometric structure, so it can explain most of the experimental phenomena [40]. However, compared with the sphere PDM, this model is more complex, and the curvature radius of the probe top needs to be selected and determined to be compared with the experimental data $[41,42]$. In view of the shortcomings of the above models, this paper proposes an improved PDM on the basis of the PDM. This improved model still uses the PDM in the form of metal sphere. However, by adjusting the radius of the sphere and the distance index of the metal sphere varying with the height of the sample, it can express the interaction function between the sample and the probe with any dielectric constant and any shape [43]. By using the metal sphere, the improved PDM can help us understand the scattering properties and imaging resolution of the probe by comparing the radius of the metal sphere, which can provide a judgment basis for the design of the probe.

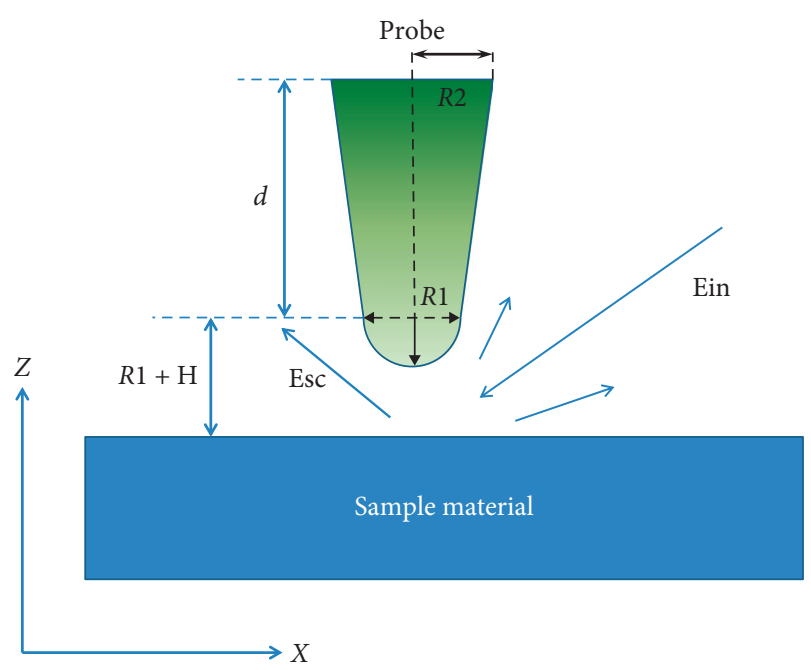

FIGURE 1: Incident and scattering fields of the near-field probe on the surface of the plane sample after incident by plane wave.

2.1. Equivalent Relation of Polarizability between Metal Sphere and Dielectric Sphere. In scanning near-field imaging, the induced dipole moment in the probe can be described by the polarizability and incident field. The scattering field has a proportional relationship with the polarizability of the probe and the incident field $[12,34,37]$ :

$$
\vec{E}_{\mathrm{sca}} \propto \vec{P}=\alpha \vec{E}_{0}
$$

It can be seen from formula (1) that the scattering field is proportional to the polarizability of the near-field probe. Therefore, in order to improve the scattering efficiency of the near-field probe, it is necessary to improve the polarizability of the near-field probe. The polarizability of the near-field probe is closely related to the geometry and the material parameters of the probe. In order to further understand the scattering properties of the near-field probe and its interaction with the sample, the analytical solutions of typical problems can be studied first. For spherical objects, the scattering analytical solutions of spheres are widely used in the literature to study the electromagnetic scattering of tiny objects. For electromagnetic scattering in SNOM subwavelength imaging, the dipole model with analytic form is widely used to analyze the interaction between the probe and the sample [39, 44-51]. For the interaction between the spherical scatterer and the plane sample, the induced dipole moment in the sphere can be approximated by

$$
P=\alpha\left\{E_{\mathrm{inc}}+E_{\mathrm{img}}(r+d)\right\}=\alpha\left\{E_{\mathrm{inc}}+\frac{\beta P}{2 \pi[2(r+d)]^{3}}\right\},
$$

where the $E_{\text {inc }}$ and $E_{\text {img }}$ are the incident field and image filed at the sphere location, respectively.

The effective polarizability of the dielectric sphere can be expressed as [43]

$$
\alpha_{\text {effpd }}=\frac{\alpha}{1-\left(\alpha \beta /\left(16 \pi(R+H)^{3}\right)\right)},
$$


where $\alpha$ is the polarizability of the dielectric sphere in free space, which can be expressed as $[29,37]$

$$
\alpha=4 \pi \varepsilon_{0} \frac{\varepsilon_{r}-1}{\varepsilon_{r}+2} R^{3} .
$$

In formula (4), $R$ is the radius of the dielectric sphere and $\varepsilon_{r}$ is the dielectric constant of the dielectric sphere. The normalized polarizability of the dielectric sphere can be expressed as

$$
\alpha_{n}=\frac{\alpha}{\varepsilon_{0} V}=3 \times \frac{\varepsilon_{r}-1}{\varepsilon_{r}+2} .
$$

For the metal sphere, its polarizability and normalized polarizability can be, respectively, expressed as [34]

$$
\begin{aligned}
\alpha & =4 \pi R^{3}, \\
\alpha_{n} & =3 .
\end{aligned}
$$

The relationship between the polarization vector of the dielectric sphere and the incident field can be expressed as

$$
P=-4 \pi \varepsilon_{0} \frac{\varepsilon_{r}-1}{\varepsilon_{r}+2} R^{3} E_{0} .
$$

It can be seen from the above formula that, when the dielectric constant of the dielectric sphere is large enough, its polarizability will be very close to that of the metal sphere. Therefore, when studying the near-field scattering problems, the dielectric sphere with a large dielectric constant can be equivalent to the metal sphere in terms of polarizability. Therefore, in order to get the equivalence relation between them, we set that the polarization formula of the metal sphere is equal to that of dielectric sphere, and then, we can get the following equation:

$$
4 \pi R_{\mathrm{diel}}^{3} \frac{\varepsilon_{r}-1}{\varepsilon_{r}+2}=4 \pi R_{\mathrm{PEC}}^{3} \longrightarrow R_{\mathrm{PEC}}^{3}=R_{\mathrm{diel}}^{3} \frac{\varepsilon_{r}-1}{\varepsilon_{r}+2} .
$$

It can be seen from equation (8) that, for a dielectric sphere with a specified radius, a metal sphere with an equivalent radius can be found corresponding to it, and they have the same polarizability. And on this basis, we will extend this conclusion further. The probe with arbitrary dielectric constant and arbitrary shape is equivalent to a metal sphere with an effective radius $R$, and they can have the same polarizability. Therefore, the near-field scattering problem can be studied by the equivalent metal sphere. To generalize the improved PDM to probes with arbitrary material parameters and shapes, the polarization of spherical probes was first calculated by the finite element method, and the results were compared with the analytical results to verify the validity of the numerical calculation method for the polarization of probes. In the finite element calculation, the static field method is adopted for the very small size of the probe. In the static field calculation, because the electric field has singularity under the condition of very small radius of curvature, the mesh is divided by $1 / 5$ of the sphere radius. The finite element method of the electrostatic field is used to calculate the polarizability of the dielectric sphere, as shown in the following equation:

$$
\begin{aligned}
& P=\varepsilon_{0} \iiint\left(\varepsilon_{r}-1\right) \cdot E \cdot \mathrm{d} V, \\
& \alpha=\frac{P}{E_{0}} .
\end{aligned}
$$

Figure 2 shows the relationship between the normalized polarizability and the dielectric constant of a dielectric sphere with different dielectric constants and radius $R$. The calculated results are consistent with the analytical results, indicating that the accuracy of the numerical calculation meets the requirements of the analysis. The same settings are used in the following finite element analysis. As can be seen from Figure 2, the normalized polarizability of dielectric sphere will approach that of metal sphere with the increase of dielectric constant. When the relative dielectric constant of the dielectric sphere is 100 , the normalized polarizability reaches 2.94 , which is very close to the normalized polarizability 3 of the metal sphere.

In order to study the equivalent relationship between the polarizability of the metal sphere and the dielectric sphere, according to formula (8), the relationship between the dielectric constant of the dielectric sphere and the equivalent radius of the metal sphere is shown in Figure 3 after fixing the radius of the dielectric sphere. As shown, it can be seen that the dielectric constant of the dielectric sphere can be uniquely equivalent to the polarizability of an equivalent radius metal sphere. In this way, the polarizability of the dielectric sphere can be measured by the equivalent radius of the metal sphere, thereby obtaining near-field scattering properties. In addition, it can also be seen from Figure 3 that the smaller the dielectric constant, the smaller the equivalent radius, and therefore, the smaller the value of the polarizability, because the polarizability of the metal sphere increases with the increase of the radius. Under the condition of the same polarizability, the dielectric spheres with fixed radius $R$ and different dielectric constants can be equivalent to the metal spheres with equivalent radius. The smaller the dielectric constant of the dielectric sphere is, the smaller the equivalent radius of the metal sphere will be. Figure 3 shows the functional relationship between the dielectric constant of the dielectric sphere with a fixed radius of 50 nanometers and the equivalent radius of the metal sphere. It can be seen that, as the dielectric constant increases, the radius of the metal sphere increases correspondingly and finally approaches the radius of the dielectric sphere.

\subsection{PDM of Metal Sphere and Dielectric Sphere in the Presence} of Samples. The effective polarizability of a metal sphere in free space is only related to the radius of the sphere. However, in the presence of samples, due to the interaction between the metal sphere and the sample, the effective polarizability is not only related to the radius of the metal sphere but also related to the distance from the sample plane and the dielectric constant of the sample [52]. For dielectric spheres in free space, the effective polarizability depends on the radius of the sphere and the dielectric constant of the sphere. In the presence of samples, due to the interaction 


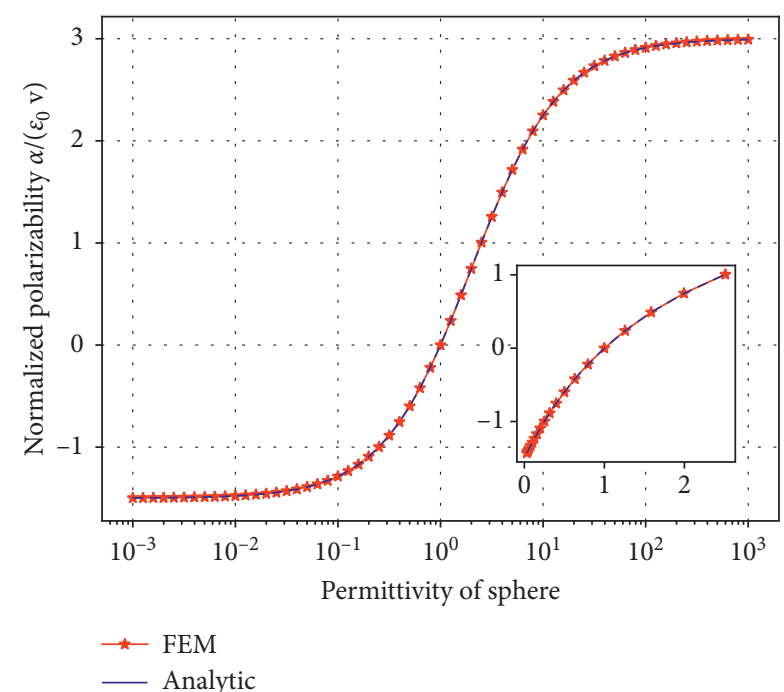

FIGURE 2: Relation curve between dielectric constant and normalized polarity of dielectric sphere with radius $R$.

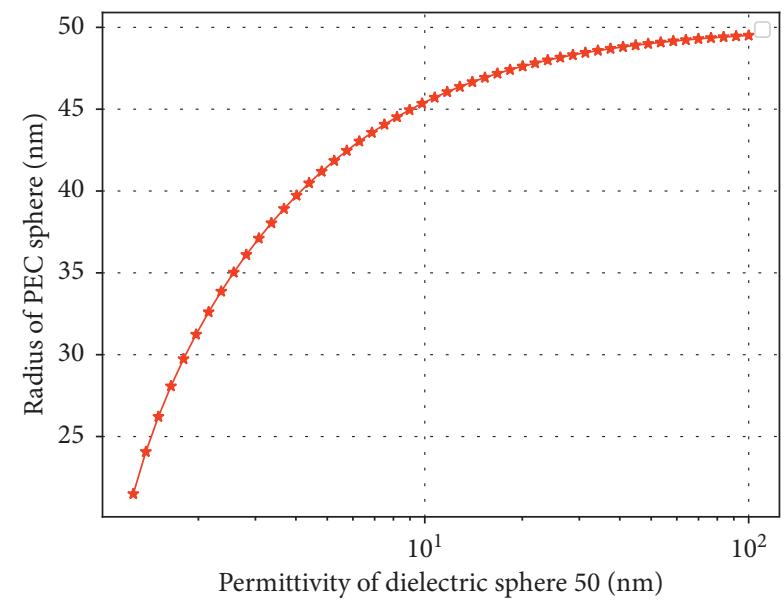

FIGURE 3: Relationship between the different dielectric constants of the dielectric sphere $(R=50 \mathrm{~nm})$ and the equivalent radius of the metal sphere.

between the dielectric sphere and the sample, the effective polarizability is not only related to the radius of the sphere but also related to the distance from the plane of the sample and the dielectric constant of the sample and the sphere.

The interaction between the probe and sample material needs to be considered in the near-field microscopy imaging technology [42, 43, 52-62], and PDM can well describe the interaction between the spherical probe and sample. Under the condition of the existence of the sample plane, the nearfield scattering can be expressed as

$$
E_{\mathrm{sca}} \propto \frac{\alpha(1+\beta)}{1-\left(\alpha \beta /\left(16 \pi(R+H)^{3}\right)\right)} E_{\mathrm{inc}}=(1+\beta) \cdot \alpha_{\mathrm{effpd}} \cdot E_{\mathrm{inc}} .
$$

In formula (10),

$$
\beta=\frac{\varepsilon_{s}-1}{\varepsilon_{s}+1},
$$

where $\varepsilon_{s}$ is the relative dielectric constant of the sample. It can be seen from formula (10) that if the dielectric spherical probe is replaced by a metal spherical probe with an equivalent radius, the scattering field or effective polarizability will be the same. Figure 4 shows the polarizability curves of the dielectric spherical near-field probe and equivalent radius metal spherical probe with the distance from the sample surface, and it can be seen that they are consistent. The results in Figure 4 show that the subwavelength imaging of the near-field probe can be studied by replacing the dielectric sphere with a metal sphere with an equivalent radius. In Figure 4, the dielectric constant of the dielectric sphere is 100 , the radius is $50 \mathrm{~nm}$, and the equivalent radius of the metal sphere is $49.5 \mathrm{~nm}$. For the PDM of dielectric spheres with other dielectric constants, the polarization PDM formula of metal spheres with an equivalent radius can get similar results.

\subsection{Improved PDM Formula for Probe with Arbitrary Shape} and Material Parameters. The effective polarizability of the probe is related to the geometry and dielectric constant of the probe [63-65].

For near-field probes with arbitrary shape and material parameters, the PDM formula of a simple spherical probe cannot be used to accurately analyze the interaction between the near-field probe and the sample. The finite dipole model can usually get more accurate analysis results, but the calculation formula is more complex. In order to apply the point-like dipole model to the analysis of near-field probes in general cases, considering that for nonspherical near-field probes, the relation with the distance from the sample will be different from that of the spherical near-field probes when the probe approaches the sample plane, so the relation exponent $n$ with the change of distance is taken as the undetermined parameter. In addition, the size of the nearfield probe has a great influence on the polarizability, so the sphere radius is also taken as an undetermined parameter.

In view of the above considerations, the effective polarizability of the improved PDM based on the metal sphere is shown in the following formula:

$$
\alpha_{\text {effpd }}(R, n)=\frac{4 \pi R^{n}}{1-\left(4 \pi R^{n} \beta /\left(16 \pi(R+H)^{n}\right)\right)}[L]^{3} .
$$

In formula (12), since the exponential $n$ of the sphere radius $R$ is an undetermined parameter, the dimension of polarizability does not meet the dimensional requirements. Therefore, in the calculation of formula (12), the value of the length variable is scaled with the length unit, and the final result is expressed in the cubic form of the corresponding length unit. In formula (12), $R$ is the radius of the equivalent sphere and $n$ is the parameter related to the polarization property of the sphere and the interaction with the sample. For the nonspherical probe, $R$ and $n$ can be obtained through optimization fitting of the polarization curve of the model and numerical calculation. Due to the universality of 


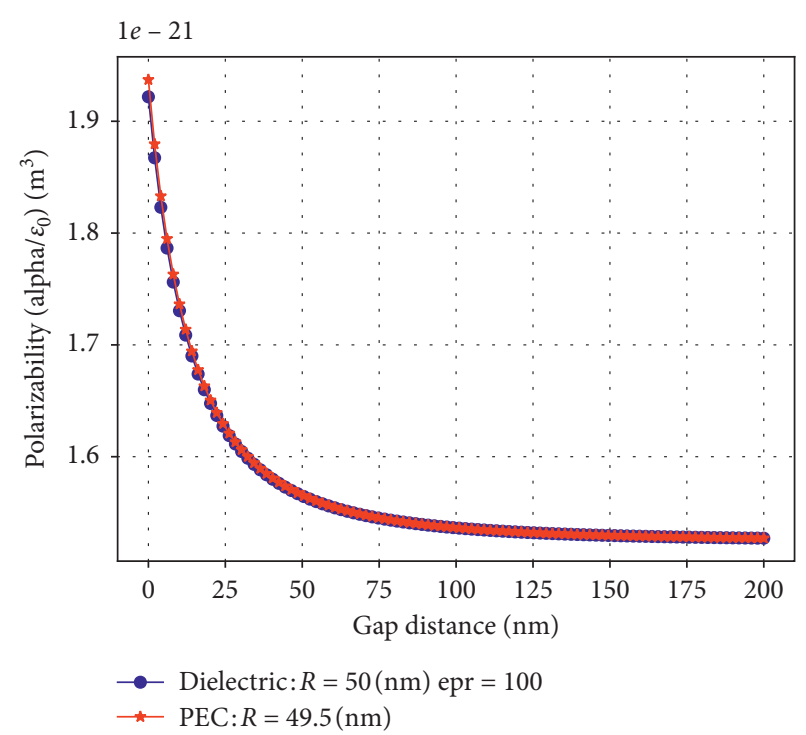

FIGURE 4: Relationship between the polarizability of the radius $50 \mathrm{~nm}$ dielectric sphere with dielectric constant 100 and the radius $49.5 \mathrm{~nm}$ metal sphere and the distance from the surface of the sample.

numerical calculation, near-field probes of any shape and material can be obtained by computational electromagnetics. On the basis of numerical calculation, the parameters of the improved PDM are optimized, which can be used for near-field scattering analysis. In order to verify the improved model proposed, we will analyze the equivalent PDM parameters of ellipsoidal and conical probes, respectively, and analyze the relationship between the equivalent model parameters and the shape and material parameters of near-field probes. Table 1 gives the cases of the study, which demonstrate the equivalence between IPDM and the SNOM probes with different geometries and permittivities.

\subsubsection{Equivalent PDM Parameters of the Ellipsoid.} Ellipsoidal near-field probe is often used as an analytical model in near-field scattering analysis. The finite dipole model (FDM) is an analytical model of the near-field probe based on ellipsoid analysis [66-68]. In order to analyze the scattering properties of the near-field probe by using formula (12), the interaction model between the near-field probe and the sample plane dielectric is analyzed for two different sizes of dielectric ellipsoids, respectively. The semishort axis of the two dielectric ellipsoids is $50 \mathrm{~nm}$, and the semilong axis is $100 \mathrm{~nm}$ and $150 \mathrm{~nm}$, respectively. In order to prove that the polarizability properties of ellipsoids can be equivalent to that of spheres with equivalent radius, we first consider the equivalent polarizability relationship between dielectric ellipsoids and metal spheres when the dielectric constant of plane samples is 12.5 .

The polarizability of the dielectric ellipsoid is calculated using the finite element method by equation (9). In the finite element analysis, the electrostatic analysis model is used to solve the Laplace equation. In the finite element calculation, the boundary conditions satisfied by the unit electrostatic field were set. A cuboid was chosen as the computing area, and its side lengths were $500 \mathrm{~nm}$ in the $x$ and $y$ directions and $800 \mathrm{~nm}$ in the $z$ direction. The dielectric ellipsoid was placed in the center of the simulation area, and the dielectric constant was 100 . The reason why the dielectric constant is chosen to be 100 is that the polarization properties of the dielectric ellipsoid are close to that of the metal sphere when the dielectric constant is 100 . If we choose a larger permittivity, we need to add more unknowns to the mesh partition. The thickness of the sample is $200 \mathrm{~nm}$. The ellipsoidal polarizability is calculated by the finite element method, and the equivalent spherical radius $R$ and exponent $n$ can be obtained by the optimization method:

$$
\min \alpha_{\text {effpd }}(R, n)-\alpha_{\text {FEM }} \text {. }
$$

Figure 5 shows the polarizability curves of ellipsoids with two different sizes as a function of distance from the sample surface with a dielectric constant of 12.5. As a comparison, the polarizability curve of the equivalent metal sphere based on the improved PDM is also given by using formula (13).

2.3.2. Equivalent PDM Parameters of the Cone Probe. As an analysis of near-field scattering, the cone probe model is closer to the actual near-field probe. Since the model is closer to the actual probe, it is difficult to get the analytic solution of the analytical model. For the actual probe, a numerical analysis model can be obtained through electromagnetic simulation analysis, but the numerical analysis model is time-consuming for near-field imaging [57]. As an improved PDM, numerical model analysis and analytical model can be combined effectively, and the parameters of the improved PDM can be obtained by the optimization method only through one numerical simulation analysis. This model can improve the efficiency of near-field imaging analysis. In particular, the improved PDM can also give a good quantitative analysis for the resolution of near-field.

Generally, the cone near-field probe can be described by three geometric parameters: the top radius, the bottom radius, and the length of the cone. In a cone probe, the bottom radius is connected to a hemisphere with a radius equal to the bottom radius of the cone. Two kinds of cone probes of different sizes are used for the equivalent approximation of the cone probes. The difference between the two cone probes is the length of the cone, which is $100 \mathrm{~nm}$ and $200 \mathrm{~nm}$, respectively. The top radius of the cone is $100 \mathrm{~nm}$, and the bottom radius is $50 \mathrm{~nm}$. The permittivity of the cone is 100. In Figures 6(a) and 6(b), the dielectric constant of the planar sample is 12.5 , which is the average dielectric constant of silicon, a general semiconductor material. Figure 6(a) shows the polarizability curve of the near-field probe with a cone length of $100 \mathrm{~nm}$ as a function of the height from the surface of the sample. As a comparison, the equivalent parameter of the improved PDM obtained by the optimization method is $R=27.43 \mathrm{~nm}$ and the distance index is $n=2.78$. It can be seen from the figure that the probe model curve of the finite element method is in good agreement with the improved PDM curve. Similarly, Figure 6(b) shows the near-field probe with a cone length of 
TABLE 1: Cases of the study for the SNOM probe polarizability.

\begin{tabular}{lcc}
\hline Case & Probe types (unit: nm) & Substrate permittivity \\
\hline 1. Figure 5(a) & Ellipsoid $(c=100, b=50)$ & 12.5 \\
2. Figure 5(b) & Ellipsoid $(c=150, b=50)$ & 12.5 \\
3. Figure 6(a) & Cone probe $(r 1=50, r 2=100$, len $=100)$ & 12.5 \\
4. Figure 6(b) & Cone probe $(r 1=50, r 2=100$, len $=200)$ & 12.5 \\
5. Figure 6(c) & Cone probe $(r 1=50, r 2=100$, len $=100)$ & 5 \\
6. Figure 6(d) & Cone probe $(r 1=50, r 2=100$, len $=200)$ & 5 \\
\hline
\end{tabular}

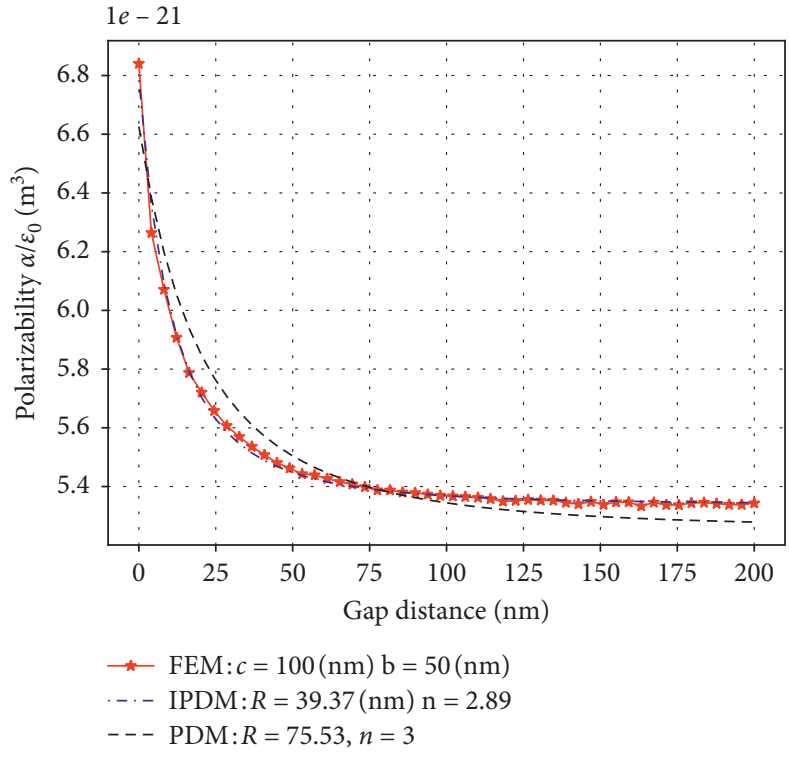

(a)

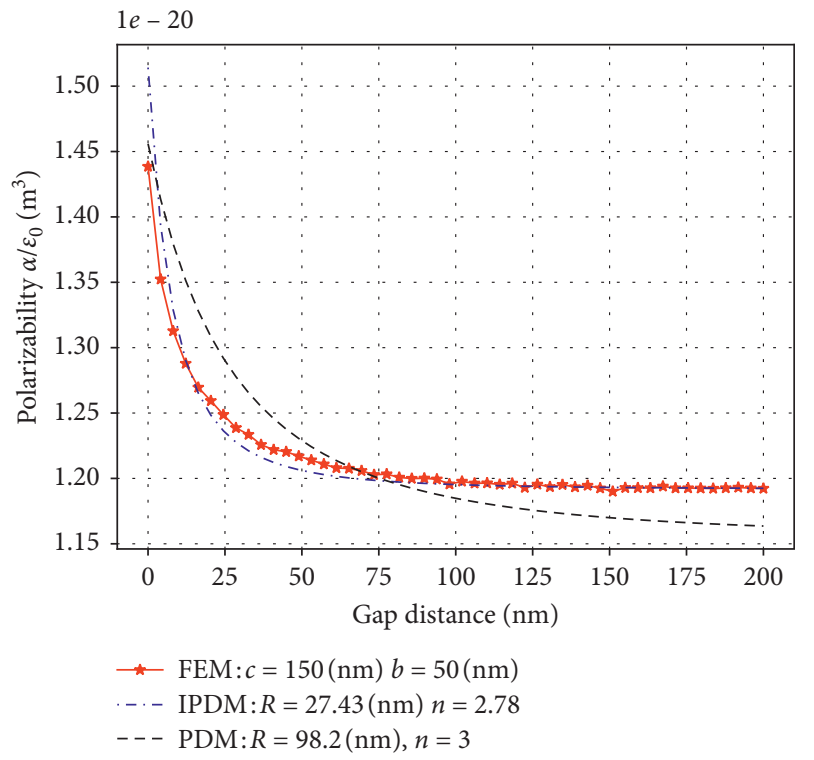

(b)

FIGURE 5: (a) The probe-approaching curve for case 1. The dielectric constant of the dielectric ellipsoid is 100 , the semiminor axis is $50 \mathrm{~nm}$, and the semimajor axis is $100 \mathrm{~nm}$. Equivalent metal sphere radius is $47.08 \mathrm{~nm}$, and distance change index $n=3.36$. (b) The probeapproaching curve for case 2 . The dielectric constant of the dielectric ellipsoid is 100 , the semiminor axis is $50 \mathrm{~nm}$, and the semimajor axis is $150 \mathrm{~nm}$. Equivalent metal sphere radius is $27.43 \mathrm{~nm}$, distance change index $n=2.78$.

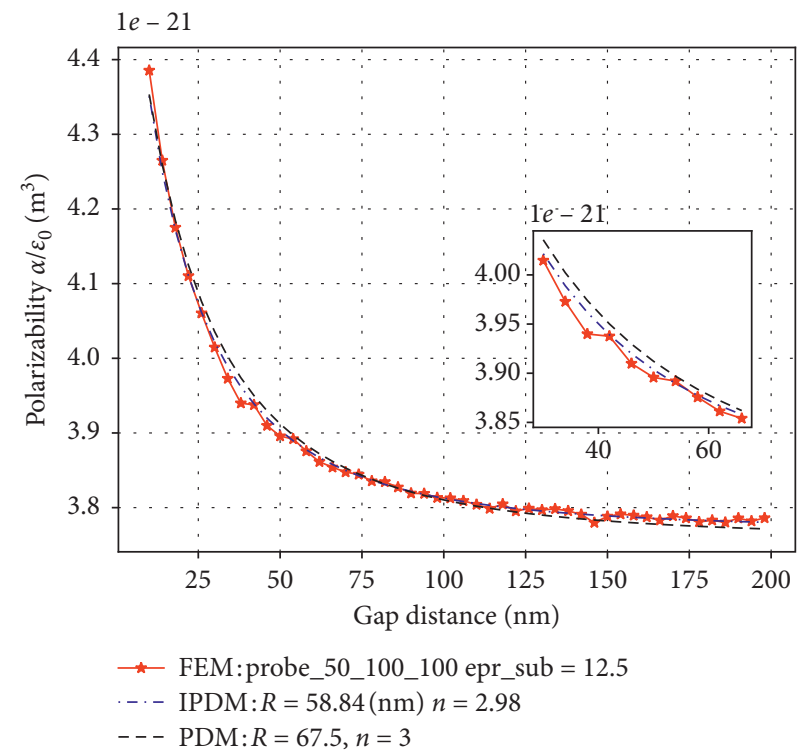

(a)

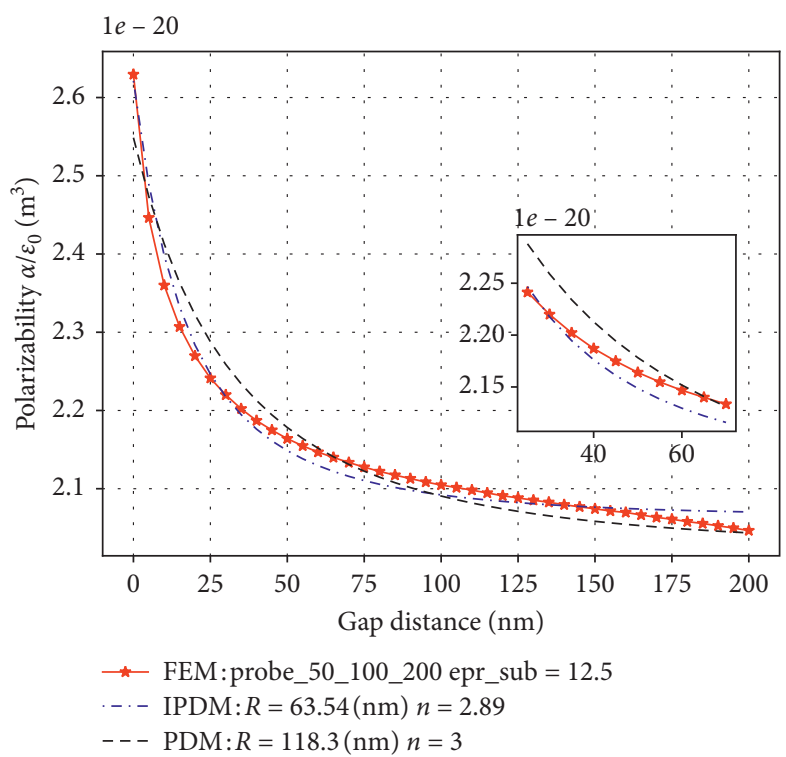

(b)

Figure 6: Continued. 


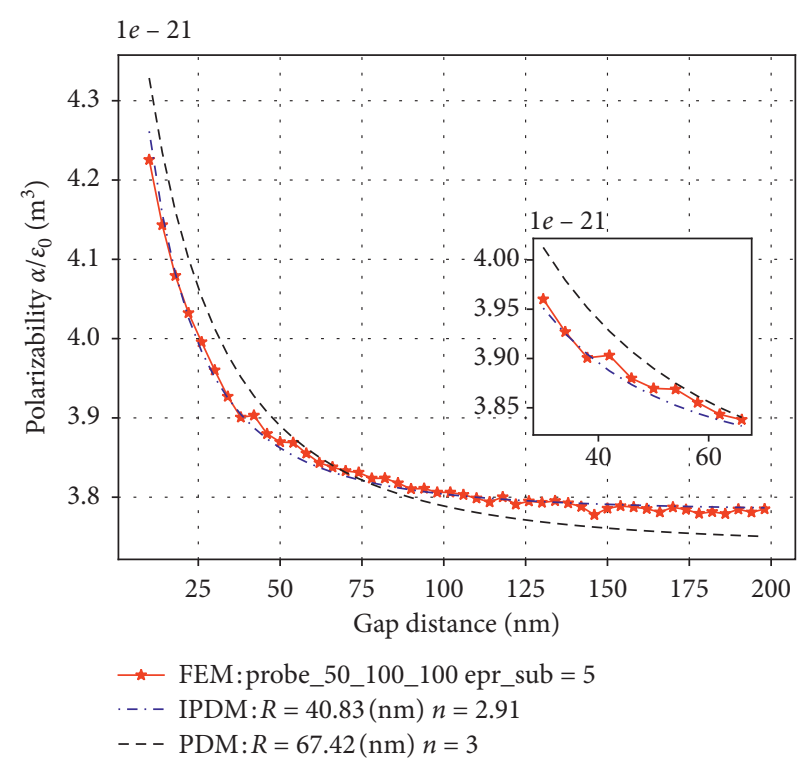

(c)

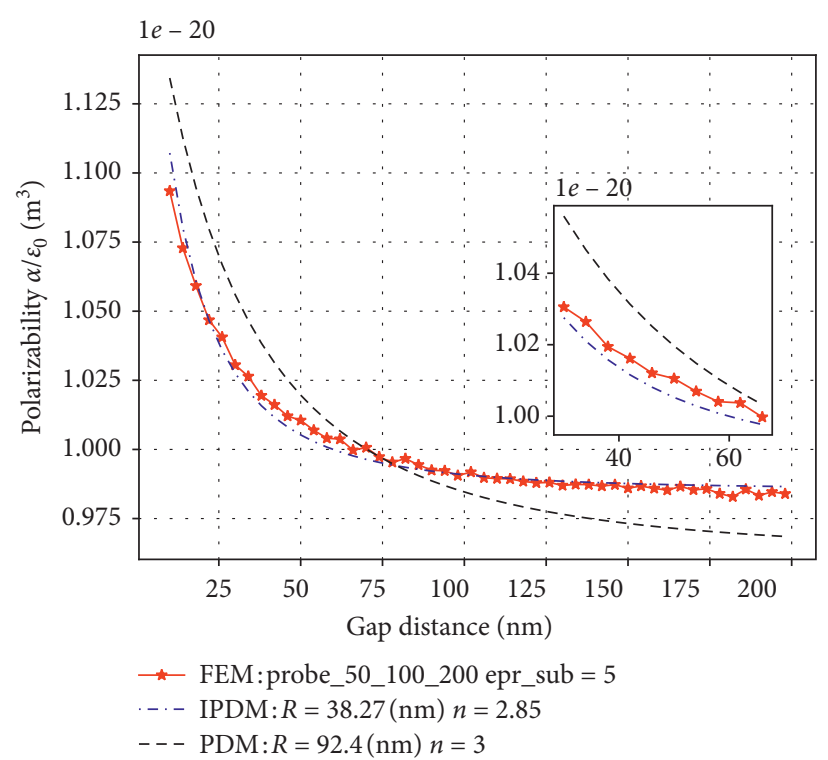

(d)

FIgURE 6: (a) The probe-approaching curve for case 3. The cone dielectric probe has a dielectric constant of 100; the cone bottom radius $R 1$ is $50 \mathrm{~nm}$, top radius $R 2$ is $100 \mathrm{~nm}$, and cone length is $d=100 \mathrm{~nm}$; an equivalent spherical radius is $R=58.84 \mathrm{~nm} ; n=2.98$. (b) The probeapproaching curve for case 4 . The cone dielectric probe has a dielectric constant of 100; the cone bottom radius is $50 \mathrm{~nm}$, top radius is $100 \mathrm{~nm}$, and cone length is $200 \mathrm{~nm}$; the equivalent spherical radius is $R=63.54 \mathrm{~nm} ; n=2.89$. (c) The probe approaching curve for case 5 . The cone dielectric probe has a dielectric constant of 100 , the cone bottom radius $R 1$ is $50 \mathrm{~nm}$, top radius $R 2$ is $100 \mathrm{~nm}$, and cone length is $d=100 \mathrm{~nm}$; the equivalent spherical radius is $R=40.83 \mathrm{~nm} ; n=2.91$. (d) The probe-approaching curve for case 6 . The cone dielectric probe has a dielectric constant of 100 , the cone bottom radius $R 1$ is $50 \mathrm{~nm}$, top radius $R 2$ is $100 \mathrm{~nm}$, and cone length is $d=200 \mathrm{~nm}$; the equivalent spherical radius is $R=38.27 \mathrm{~nm} ; n=2.85$.

$200 \mathrm{~nm}$ as a function of height from the sample. As a comparison, the equivalent parameter of the improved PDM obtained by the optimization method is $R=63.54 \mathrm{~nm}$ and the distance index is $n=2.89$. The dielectric constant of the planar sample is changed to 5. Similarly, the curves of the polarizability of the cone probe with two sizes varying with the height distance from the planar sample are calculated by the finite element method. The calculation result is shown in Figures 6(c) and 6(d), whereas in Figure 6(c), it is a plot of the polarizability of a cone of $100 \mathrm{~nm}$ length as a function of height distance. Figure 6(d) is a plot of the polarizability of a cone of $200 \mathrm{~nm}$ length as a function of height. As can be seen from the results in the figure, the improved PDM can well describe the polarizability properties of any shape near-field probe.

2.3.3. Analysis. For the dielectric ellipsoid model, the analytical solution of ellipsoid is often used as the approximation of the actual probe to analyze the model, and the ellipsoid model has better approximation than the sphere model. Here, the improved PDM method is proposed to fit the equivalent radius and distance exponent of the PDM on the basis of numerical analysis, which can quickly and accurately analyze the interaction between the near-field probe and the sample. Figures 6(a) and 6(b) are polarizability curve variation with height distance from the sample when the sample dielectric constant is 12.5 and the near-field probe approaches the sample. The length of the near-field probe in
Figure 6(a) is $100 \mathrm{~nm}$, and the length of the near-field probe in Figure 6(b) is $200 \mathrm{~nm}$. By comparing their equivalent spherical radius and distance index, it is not difficult to find that the equivalent radius of the short probe is larger than that of the long probe and the distance index of the short probe is also larger than that of the long probe. Figures 6(c) and $6(\mathrm{~d})$ are plots of the distance-dependent change of the near-field probe as it approaches the sample with a dielectric constant of 5. Similarly, in Figures 6(c) and 6(d), the equivalent radius of the short probe is larger than that of the long probe; the distance index of the short probe is larger than that of the long probe. In the following study of nearfield contrast, the equivalent sphere radius can be used as a parameter to measure the resolution, so a small equivalent radius means a higher resolution. The distance index reflects the interaction between the near-field probe and the sample, while a small distance index reflects that the interaction changes slowly with the increase of distance; it is therefore possible to observe the contrast at a higher position from the sample.

\section{Resolution and Contrast Analysis of Near- Field Scanning}

In optical microscopic imaging, contrast is often used to describe the transition width of transition curve shapes at different material boundaries of samples as the definition of spatial resolution. Such shape of transition curve is usually called edge response function (ERF). The characteristic 
width of ERF is obtained by its derivative, also called (line spread function) curve transition width $w$. In scattered-type SNOM, $w$ can be obtained by direct measurement or by its derivative. However, for the boundaries of different materials with different heights, it is very important to estimate $w$ effectively due to the convolution of the probe and sample. Figure 7 shows the schematic diagram of near-field probe scanning.

In order to quantitatively estimate the resolution of SNOM, in this paper, the improved PDM is proposed to quantitatively study the transition curves of sample surfaces with different heights or different permittivities by using equivalent spheres. In addition, the transition curves of samples with discontinuous permittivity and discontinuous height are also quantitatively studied. The basic idea of describing the shape of transition curve by the radius of equivalent spherical is that if the sphere is located at the interface of two samples with different properties, the equivalent dielectric constant or equivalent height of the sample can be considered as the arithmetic average of material properties on both sides. Considering the case where the distance between the center of the sphere and the interface is larger than the radius of the sphere, it can be regarded as equivalent to the infinite plane; the dielectric constant of the sample at the boundary is regarded as the average of the dielectric constants on both sides of the interface. According to the continuous transition property of the function, a transition function in the form of an exponent is introduced. Finally, the equivalent dielectric constant of the sample can be expressed as

$$
\varepsilon_{r}=\frac{\varepsilon_{r, R}+\varepsilon_{r, L} \times e^{-\left(\left(x-x_{0}\right) / R\right)}}{1+e^{-\left(\left(x-x_{0}\right) / R\right)}},
$$

where $x_{0}$ is the interface position, $R$ is the radius of the equivalent sphere, $\varepsilon_{r, L}$ is the dielectric constant of the side where $x$ is less than the interface position, and $\varepsilon_{r, R}$ is the dielectric constant of the side where $x$ is greater than the interface position. Similarly, if the heights of both sides of the interface are different, then the equivalent height of the dielectric can be expressed as

$$
h=\frac{h_{R}+h_{L} \times e^{-\left(\left(x-x_{0}\right) / R\right)}}{1+e^{-\left(\left(x-x_{0}\right) / R\right)}} .
$$

The scattering near-field is calculated by formula (10).

In Figure 8, contrast is calculated using contrast transition functions, which are related to the equivalent spherical radius of IPDM. In other words, the equivalent radius of the metal sphere is used to describe the resolution of SNOM. The smaller the equivalent spherical radius is, the higher the resolution is. Table 2 gives the cases of the study, which demonstrate the scattering near-field contrast curves of samples based on IPDM.

Figure 8(a) shows the contrast curves of near-field scattering of samples with different dielectric constants. According to the previous analysis, if the dielectric constant of the sample is different, the equivalent metal sphere radius $R$ and distance exponent $n$ of the improved PDM will be different. In order to analyze the contrast curves of different

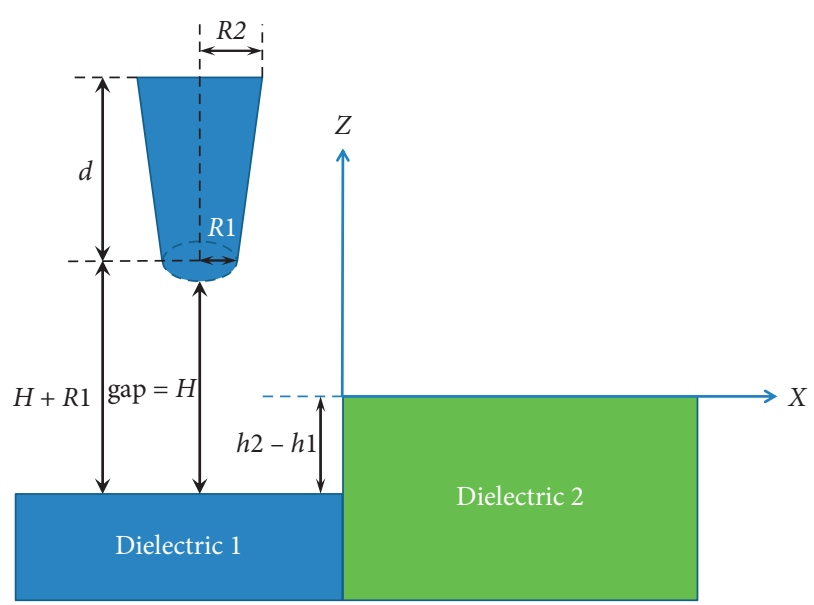

FIGURE 7: Schematic diagram of near-field probe scanning with sample surfaces of different structures and the probe scanning along the $x$ direction.

dielectric constants, it is necessary to consider the function relationships between the equivalent spherical radius and the distance exponent as the scanning distance. The function of radius $R$ and distance exponent $n$ with scanning distance $x$ are defined as

$$
\begin{aligned}
& R(x)= \begin{cases}R_{1}, & x<x_{0}, \\
R_{2}, & x>x_{0},\end{cases} \\
& n(x)= \begin{cases}n_{1}, & x<x_{0}, \\
n_{2}, & x>x_{0} .\end{cases}
\end{aligned}
$$

The results of equation (16) are substituted into the improved PDM to obtain the near-field transition curve. It can be seen from Figure 8(a) that the transition curves obtained by the improved PDM and the finite element calculation model are in good agreement. Meanwhile, the resolution of the probe tip can be given quantitatively by the equivalent sphere radius.

Figure 8(b) shows the near-field transition curve of the sample plane with the same dielectric constant and different heights. The dielectric constant of the sample is 12.5 . The height difference between the two sides of the interface is $h 2-h 1=50 \mathrm{~nm}$. In the area $x<0$, the distance between the near-field probe and the sample surface is $50 \mathrm{~nm}$; in the area $x>0$, the distance between the near-field probe and the sample surface is 0 . It can be seen from Figure $8(b)$ ) that the simulation results of the improved PDM and FEM are in good agreement.

Figure 8(c) shows the contrast curves of near-field scanning with different permittivities and heights on both sides of the dividing line. According to Figure 8(a), it can be seen that the sample with a large dielectric constant will generate a larger scattering field. As can be seen from Figure $8(\mathrm{~b})$, the closer the probe is to the sample surface, the stronger the scattering field is. In Figure 8(c), we consider that the area on the right side of the dividing line which has a small dielectric constant and a small distance between the probe and the sample surface. The results show that there is a larger scattering field on the right side of the interface, 


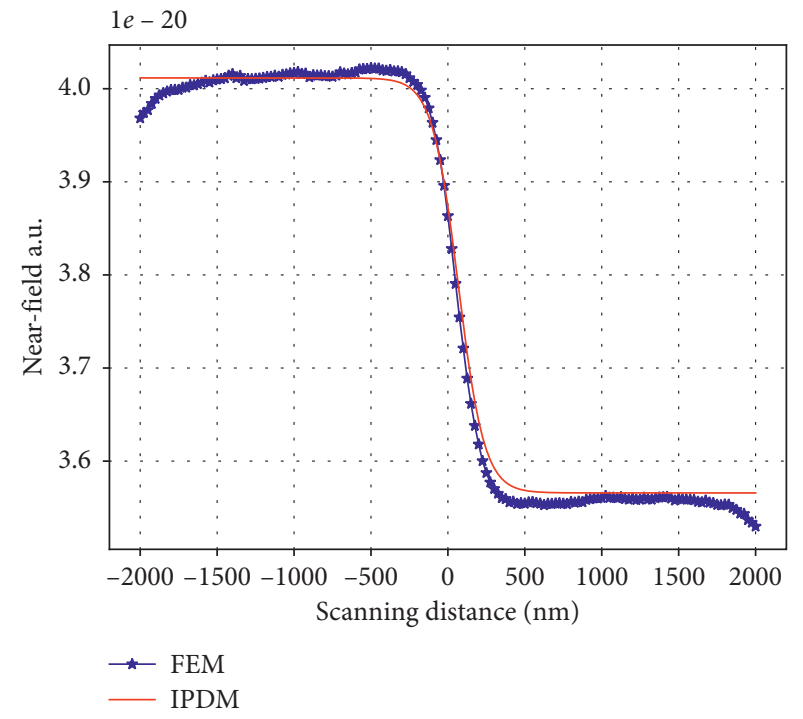

(a)

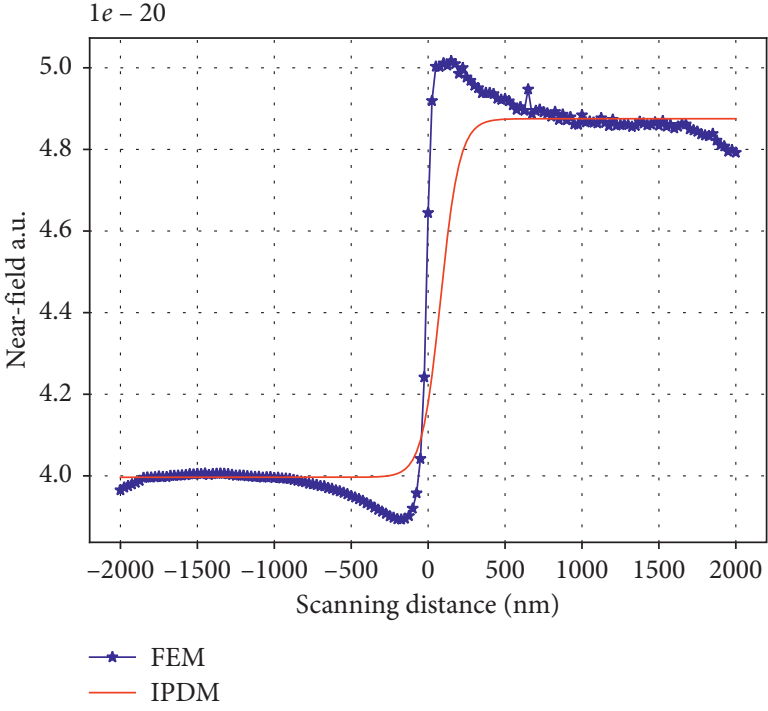

(b)

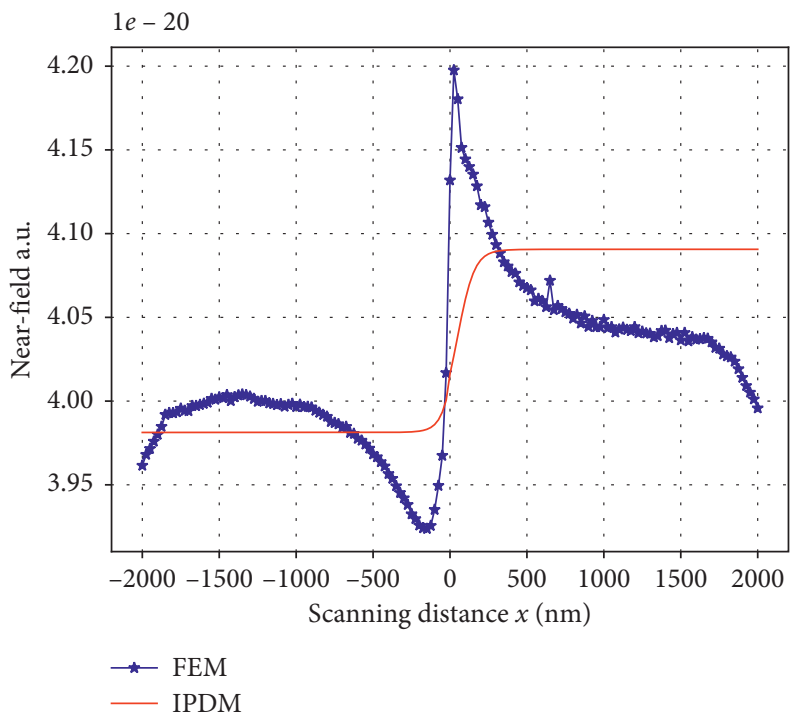

(c)

FiguRE 8: (a) Scattering near-field contrast curves of samples with different dielectric constants on both sides of the dividing line. The dividing line is $x=0$. (b) Scattering near-field contrast curves of samples with different heights on both sides of the dividing line. The dividing line is located at $x=0$. (c) Near-field contrast curves with different dielectric constants and heights on both sides of the sample dividing line. The dividing line is at $x=0$.

TABLE 2: Cases of the study for the scattered near-field contrast curves of samples (unit: $\mathrm{nm}$ ).

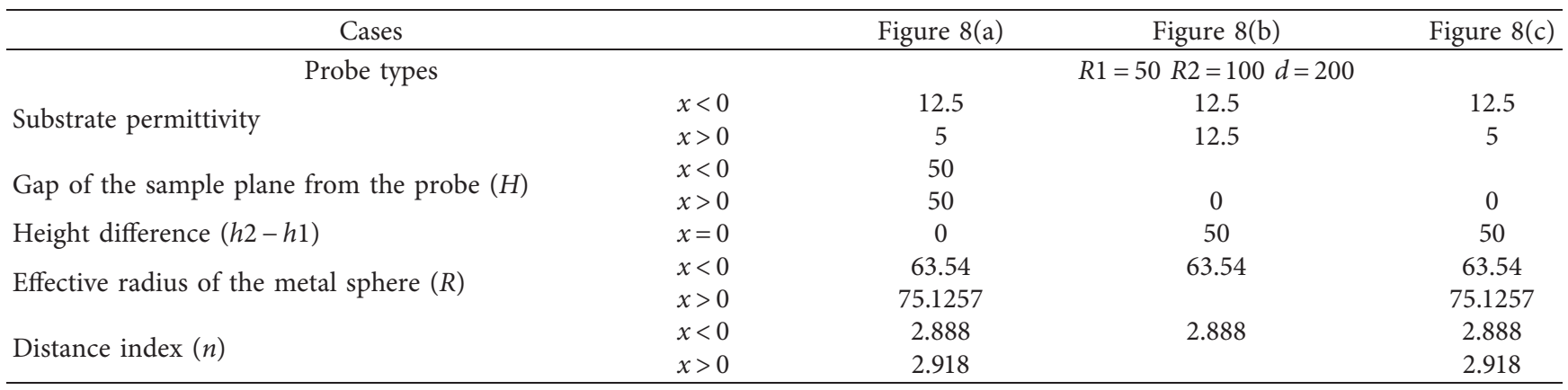


indicating that the height has a greater influence on the contrast of the scattering field.

3.1. Analysis. The surface of the sample has different structural properties, which will form a transition curve in the near-field scanning imaging. The steepness of the transition curve reflects the resolution of subwavelength imaging. At present, the derivative of transition curve is usually used to describe the resolution of near-field imaging in the studies of contrast [58]. In this paper, the equivalent spherical radius is used to describe the steepness of the transition curve, and therefore, the resolution of near-field imaging is measured by this parameter value. Figure 8(a) shows the contrast curves of different dielectric constants on both sides of the sample, and the results of finite element simulation are consistent with those of the improved PDM method. Figure 8(b) shows the contrast curve with the same dielectric constant on both sides of the sample and a height difference of $50 \mathrm{~nm}$. The finite element simulation results are generally consistent with the improved PDM method. At the discontinuous interface with the height of the sample, the contrast curve of near-field scattering obtained by FEM has an overshoot waveform near the boundary due to the abrupt change of the electric field. In general, the actual sample surface is gradual rather than drastically changed. The change of the sample surface height shown here is only an extreme case; for the actual sample surface, the overshoot phenomenon will be very weak, so the agreement of contrast curve obtained by FEM will be better with the improved PDM results. Figure 8(c) shows the case where the dielectric constant and height are not continuous on both sides of the interface. Similarly, due to the discontinuous change of height, there are overshoot waveforms on both sides of the interface. In addition, the curve shape has a large deformation due to the discontinuity of the dielectric constant.

\section{Discussion and Conclusion}

In scanning near-field optical microscopy, it is very important to study the interaction and scattering mechanism between the near-field probe and the sample to understand the imaging mechanism and analyze the structural and physical properties of the sample $[59,60,63]$. In the study of near-field scattering, the analytical method has the advantage of clear physical concepts, but it cannot be used for complex shape objects $[4,69,70]$. The advantage of numerical method is that it can deal with arbitrary shape structure, but it usually consumes a lot of computing time and resources [52]. This paper extends the research based on the PDM. First, the interaction between the arbitrary nearfield probe and sample is studied by the numerical method. Then, the PDM is extended, and the improved PDM has similar analytical expression in form, but the equivalent metal sphere radius $R$ and the distance index $n$ are different from those of the original spherical PDM formula. These two parameters are obtained by the optimization method. Under the condition of equivalent polarizability, two parameters of the improved PDM are obtained by the fitting method according to application conditions.

In order to verify the feasibility of the improved PDM, the equivalents of dielectric spheres and dielectric ellipsoids with different shapes and sizes in free space and their equivalents with the improved PDM were studied. On this basis, the equivalence of dielectric sphere, ellipsoid, general shape probe, and metal sphere of the improved PDM is further studied. The results show that the SNOM probe can be composed of different materials, which show different electromagnetic response characteristics in different frequency bands. Probes of various materials and shapes can be equivalent to spherical-like structures. The meaning of spherical-like probe indicates that the dipole model is derived from the interaction model between the metal sphere and sample, which is an improvement on the basis of PDM. The difference is that the power of the sphere radius is not a traditional cubic relation, but a real number around 3, which can be obtained by the fitting method according to the interaction curve between the probe and the sample. The main advantage of the improved model is that simple PDMlike formulas can be used to analyze the microscopic imaging of SNOM. At the same time, it can give us a standard to compare the scattering characteristics of different probes because probes of arbitrary shape and material characteristics can come down to the power of the radius of the equivalent sphere and the radius of the equivalent sphere. These two parameters can provide us with the resolution of microscopic imaging and the optimal detection distance, respectively.

In the scanning near-field optical microscopy, the contrast and resolution of different surfaces are the key technical indicators [71-73]. Nevertheless, to date, most papers mainly focus on the contrast analysis of different materials and the contrast analysis of different heights. Few papers consider the two factors of different materials and different heights together. Here, we consider the influence of different materials and different heights on the near-field scattering contrast together. First, the transition curve formulas of height transition and material parameter transition between different regions are given, respectively. For the planar samples with different heights, the height of the probe on both sides of the dividing line is different. Considering the equivalent sphere radius of the probe, when the equivalent sphere of the probe is on a certain side, the distance between the probe and the sample is the height of this position. On both sides of the interface, the effective height perceived by the probe is a gradual height. Based on the above consideration, formula (15) is proposed as the height of the probe above the interface. In formula (15), on the left side of the interface, the exponential function tends to infinity with the increase of $x$ and the height value is $h_{2}$. At the interface, the exponential function is 1 and the height is $\left(h_{1}+h_{2}\right) / 2$. On the right side of the interface, with the increase of $x$, the exponential function tends to zero and the height tends to the height value of the right plane. Similarly, considering the interface of different materials, the transition curves of material parameters are given. The transition curve of the interface can be obtained by substituting the 
transition curve of height and material parameters into the IPDM formula. For the linear shape of the contrast curve of the transition region in contrast, we proposed that the equivalent sphere radius of the IPDM is used as the transition parameter of the exponential curve and the calculated transition curve is in good agreement with the numerical results.

\section{Data Availability}

No data were used to support the study.

\section{Conflicts of Interest}

The authors declare that they have no conflicts of interest.

\section{Acknowledgments}

This work was supported by the Science and Technology on Electromagnetic Scattering Laboratory, under Grant no. 621802 Y030204.

\section{References}

[1] H. A. Huckabay, K. P. Armendariz, W. H. Newhart et al., "Near-field scanning optical microscopy for high-resolution membrane studies," Methods in Molecular Biology, vol. 950, 2012.

[2] Z. H. Kim and S. R. Leone, "High-resolution apertureless near-field optical imaging using gold nanosphere probes," The Journal of Physical Chemistry B, vol. 110, no. 40, pp. 1980419809, 2006.

[3] A. Jarzembski, C. Shaskey, and K. Park, "Review: tip-based vibrational spectroscopy for nanoscale analysis of emerging energy materials," Frontiers in Energy, vol. 12, no. 1, pp. 43-71, 2018.

[4] V. E. Babicheva, S. Gamage, M. I. Stockman, and Y. Abate, "Near-field edge fringes at sharp material boundaries," Optics Express, vol. 25, no. 20, pp. 23935-23944, 2017.

[5] J. Chen, M. Badioli, and P. Alonso-González, "Optical nanoimaging of gate-tunable graphene plasmons," Nature, vol. 487, no. 7405, p. 751, 2012.

[6] Z. Fei, A. S. Rodin, W. Gannett et al., "Electronic and plasmonic phenomena at graphene grain boundaries," Nature Nanotechnology, vol. 8, no. 11, pp. 821-825, 2013.

[7] A. Woessner, P. Alonso-González, M. B. Lundeberg et al., "Near-field photocurrent nanoscopy on bare and encapsulated graphene," Nature Communications, vol. 7, p. 10783, 2016.

[8] G. X. Ni, A. S. Mcleod, Z. Sun et al., "Fundamental limits to graphene plasmonics," Nature, vol. 557, no. 7706, pp. 530533, 2018.

[9] H. T. Chorsi and J. X. J. Zhang, "Apertureless near-field scanning probes based on graphene plasmonics," IEEE Photonics Journal, vol. 9, no. 1, Article ID 4800207, 7 pages, 2017.

[10] S. Usui, S. Kitade, F. Kohmura, K. Kensuke, F. Kusa, and S. Ashihara, "Near-field imaging of infrared nanoantenna modes under oblique illumination," The Journal of Physical Chemistry C, vol. 121, no. 46, pp. 26000-26006, 2017.

[11] S. Mastel, M. Lundeberg, P. Alonso-Gonzalez et al., “THz nano focusing with cantilevered THz-resonant antenna tips," Nano Letters, vol. 17, no. 11, pp. 6526-6533, 2017.
[12] M. Almajhadi and H. K. Wickramasinghe, "Contrast and imaging performance in photo induced force microscopy," Optics Express, vol. 25, no. 22, pp. 26923-26938, 2017.

[13] A. A. Govyadinov, I. Amenabar, F. Huth, P. S. Carney, and R. Hillenbrand, "Quantitative measurement of local infrared absorption and dielectric function with tip-enhanced nearfield microscopy," The Journal of Physical Chemistry Letters, vol. 4, no. 9, pp. 1526-1531, 2013.

[14] D. E. Tranca, S. G. Stanciu, H. Radu, B. M. Witgen, and G. A. Stanciu, "Nanoscale mapping of refractive index by using scattering-type scanning near-field optical microscopy," Nanomedicine: Nanotechnology, Biology and Medicine, vol. 14, no. 1, pp. 47-50, 2018.

[15] A. Jarzembski, C. Shaskey, R. A. Murdick, and K. Park, "Temperature sensitivity of scattering-type near-field nanoscopic imaging in the visible range," Applied Optics, vol. 58, no. 8, pp. 1978-1983, 2019.

[16] L. Wang and X. G. Xu, "Scattering-type scanning near-field optical microscopy with reconstruction of vertical interaction," Nature Communications, vol. 6, p. 8973, 2015.

[17] H. Wang, L. Wang, D. S. Jakob et al., "Tomographic and multimodal scattering-type scanning near-field optical microscopy with peak force tapping mode," Nature Communications, vol. 9, no. 1, p. 2005, 2018.

[18] F. Armani, A. Boscolo, M. Bressanutti et al., "Development of a novel SNOM probe for in liquid biological samples," Lecture Notes in Electrical Engineering, Springer, Berlin, Germany, 2015.

[19] A. J. Ward and J. B. Pendry, "The theory of SNOM: a novel approach," Optica Acta: International Journal of Optics, vol. 44, no. 9, p. 12, 1997.

[20] J. A. Porto, P. Johansson, S. P. Apell et al., "Resonance shift effects in apertureless scanning near-field optical microscopy," Physical Review B, vol. 67, no. 8, Article ID 085409, 2003.

[21] D.-S. Kim and Z. H. Kim, "Role of in-plane polarizability of the tip in scattering near-field microscopy of a plasmonic nanoparticle," Optics Express, vol. 20, no. 8, p. 8689, 2012.

[22] K. G. Lee, H. W. Kihm, K. J. Ahn et al., "Vector field mapping of local polarization using gold nanoparticle functionalized tips: independence of the tip shape," Optics Express, vol. 15, no. 23, pp. 14993-15001, 2007.

[23] L. Sun, B. Bai, X. Meng, T. Cui, G. Shang, and J. Wang, "Nearfield probing the magnetic field vector of visible light with a silicon nanoparticle probe and nanopolarimetry," Optics Express, vol. 26, no. 19, pp. 24637-24652, 2018.

[24] B. Bai, J. Wang, and L. Sun, "Probing vectorial near field of light: imaging theory and design principles of nanoprobes," Optics Express, vol. 26, no. 14, Article ID 18644, 2018.

[25] B. Mourched, E. L. Nativel, R. Kribich, P. Falgayrettes, and P. Gall-borrut, "Study of light emission and collection in a transparent dielectric cantilever-based near-field optical probe," Journal of Microscopy, vol. 262, no. 1, pp. 3-11, 2016.

[26] A. G. T. Ruiter, M. H. P. Moers, and N. F. Van Hulst, "Microfabrication of near-field optical probes," Journal of Vacuum Science \& Technology B: Microelectronics and Nanometer Structures, vol. 14, no. 2, pp. 597-601, 1996.

[27] Y. Inouye and S. Kawata, "A scanning near-field optical microscope having scanning electron tunnelling microscope capability using a single metallic probe tip," Journal of $\mathrm{Mi}$ croscopy, vol. 178, no. 1, pp. 14-19, 1995.

[28] J. Sun, P. S. Carney, and J. C. Schotland, "Strong tip effects in near-field scanning optical tomography," Journal of Applied Physics, vol. 102, no. 10, p. 103103, 2007. 
[29] D. Denkova, N. Verellen, A. V. Silhanek et al., "Near-field aperture-probe as a magnetic dipole source and optical magnetic field detector," Physics, 2014.

[30] O. Huth, F. Rüting, S.-A. Biehs, and M. Holthaus, "Shapedependence of near-field heat transfer between a spheroidal nanoparticle and a flat surface," The European Physical Journal Applied Physics, vol. 50, no. 1, pp. 10603-10610, 2010.

[31] T. J. Antosiewicz, M. Marciniak, and T. Szoplik, "On SNOM resolution improvement," Photonic Crystals: Physics and Technology, Springer, Berlin, Germany, pp. 217-235, 2008.

[32] B. Paul, E. Sabastine, and F. Giovanni, "A review of threedimensional scanning near-field optical microscopy (3DSNOM) and its applications in nanoscale light management," Applied Sciences, vol. 7, no. 10, p. 973, 2017.

[33] S. Weisenburger and V. Sandoghdar, "Light microscopy: an ongoing contemporary revolution," Contemporary Physics, vol. 56, no. 2, pp. 123-143, 2015.

[34] A. Cvitkovic, N. Ocelic, and R. Hillenbrand, "Analytical model for quantitative prediction of material contrasts in scattering-type near-field optical microscopy," Optics Express, vol. 15, no. 14, pp. 8550-8565, 2007.

[35] M. Esslinger and R. Vogelgesang, "Reciprocity theory of apertureless scanning near-field optical microscopy with point-dipole probes," ACS Nano, vol. 6, no. 9, pp. 8173-8182, 2012.

[36] M. Wächter, M. Nagel, and H. Kurz, "Tapered photoconductive terahertz field probe tip with subwavelength spatial resolution," Applied Physics Letters, vol. 95, no. 4, Article ID 041112, 2009.

[37] B. Knoll and F. Keilmann, "Enhanced dielectric contrast in scattering-type scanning near-field optical microscopy," Optics Communications, vol. 182, no. 4-6, pp. 321-328, 2000.

[38] B. Knoll and F. Keilmann, "Near-field probing of vibrational absorption for chemical microscopy," Nature, vol. 399, no. 6732, pp. 134-137, 1999.

[39] M. B. Raschke and C. Lienau, "Apertureless near-field optical microscopy: tip-sample coupling in elastic light scattering," Applied Physics Letters, vol. 83, no. 24, pp. 5089-5091, 2003.

[40] A. Jarzembski and K. Park, "Finite dipole model for extreme near-field thermal radiation between a tip and planar SiC substrate," Journal of Quantitative Spectroscopy and Radiative Transfer, vol. 191, pp. 67-74, 2017.

[41] H. Nguyen Pham, S. Hisatake, O. V. Minin et al., "Enhancement of spatial resolution of terahertz imaging systems based on terajet generation by dielectric cube," APL Photonics, vol. 2, no. 5, Article ID 056106, 2017.

[42] S. C. Kehr, Y. M. Liu, L. W. Martin et al., "Near-field examination of perovskite-based superlenses and superlensenhanced probe-object coupling," Nature Communications, vol. 2, p. 249, 2011.

[43] R. Hillenbrand, B. Knoll, and F. Keilmann, "Pure optical contrast in scattering-type scanning near-field microscopy," Journal of Microscopy, vol. 202, no. 1, 2001.

[44] L. Novotny, D. W. Pohl, and B. Hecht, "Scanning near-field optical probe with ultrasmall spot size," Optics Letters, vol. 20, no. 9, pp. 970-972, 1995.

[45] Y. Liu, D. Hu, L. Pang, F. Gao, Z. Zhang, and J. Du, "Optoplasmonic probe to realize scanning near-field Raman microscopy," Optics Express, vol. 24, no. 5, pp. 5243-5252, 2016.

[46] A. E. Klein, N. Janunts, S. Schmidt et al., "Dual-SNOM investigations of multimode interference in plasmonic strip waveguides," Nanoscale, vol. 9, no. 20, pp. 6695-6702, 2017.
[47] P. Li, I. Dolado, F. J. Nikitin et al., "Optical nanoimaging of hyperbolic surface polaritons at the edges of van der Waals materials," Nano Letters, vol. 17, no. 1, pp. 228-235, 2017.

[48] R. Fikri, D. Barchiesi, F. H’Dhili et al., "Modeling recent experiments of apertureless near-field optical microscopy using 2D finite element method," Optics Communications, vol. 221, no. 1-3, pp. 13-22, 2003.

[49] M. Lucas and E. Riedo, "Invited Review Article: combining scanning probe microscopy with optical spectroscopy for applications in biology and materials science," Review of Scientific Instruments, vol. 83, no. 6, Article ID 061101, 2012.

[50] M. B. Wabuyele, M. Culha, G. D. Griffin et al., "Near-field scanning optical microscopy for bioanalysis at nanometer resolution," Methods in Molecular Biology, vol. 300, pp. 437-452, 2005.

[51] J. N. Walford, J. A. Porto, R. Carminati et al., "Influence of tip modulation on image formation in scanning near-field optical microscopy," Journal of Applied Physics, vol. 89, no. 9, pp. 5159-5169, 2001.

[52] J. M. Atkin, S. Berweger, A. C. Jones, and M. B. Raschke, "Nano-optical imaging and spectroscopy of order, phases, and domains in complex solids," Advances in Physics, vol. 61, no. 6, pp. 745-842, 2012.

[53] F. Buersgens, G. Acuna, C. H. Lang, S. I. Potrebic, S. Manus, and R. Kersting, "Shear force control for a terahertz near field microscope," Review of Scientific Instruments, vol. 78, no. 11, Article ID 113701, 2007.

[54] M. Berta, P. Kužel, and F. Kadlec, "Study of responsiveness of near-field terahertz imaging probes," Journal of Physics D: Applied Physics, vol. 42, no. 15, Article ID 155501, 2009.

[55] F. Keilmann and R. Hillenbrand, "Near-field microscopy by elastic light scattering from a tip," Philosophical Transactions of the Royal Society A: Mathematical, Physical and Engineering Sciences, vol. 362, no. 1817, pp. 787-805, 2004.

[56] R. Hillenbrand and F. Keilmann, "Complex optical constants on a subwavelength scale," Physical Review Letters, vol. 85, no. 14, pp. 3029-3032, 2000.

[57] K.-T. Lin, S. Komiyama, and Y. Kajihara, "Tip size dependence of passive near-field microscopy," Optics Letters, vol. 41, no. 3 , p. $484,2016$.

[58] S. Mastel, A. A. Govyadinov, C. Maissen, A. Chuvilin, A. Berger, and R. Hillenbrand, "Understanding the image contrast of material boundaries in IR nanoscopy reaching 5 nm spatial resolution," ACS Photonics, vol. 5, no. 8, pp. 3372-3378, 2018.

[59] K. Joulain, P. Ben-Abdallah, P. O. Chapuis et al., "Tip-sample electromagnetic interaction in the infrared: effective polarizabilities, retarded image dipole model and near-field thermal radiation detection," China Modern Educational Equipment, 2012.

[60] A. L. Lereu, A. Passian, and P. Dumas, "Near field optical microscopy: a brief review," International Journal of Nanotechnology, vol. 9, no. 3-7, p. 488, 2012.

[61] J. Gersten and A. Nitzan, "Electromagnetic theory of enhanced Raman scattering by molecules adsorbed on rough surfaces," The Journal of Chemical Physics, vol. 73, no. 7, pp. 3023-3037, 1980.

[62] P. K. Aravind and H. Metiu, "The effects of the interaction between resonances in the electromagnetic response of a sphere-plane structure; applications to surface enhanced spectroscopy," Surface Science, vol. 124, no. 2-3, pp. 506-528, 1983.

[63] A. Thoma and T. Dekorsy, "Influence of tip-sample interaction in a time-domain terahertz scattering near field 
scanning microscope," Applied Physics Letters, vol. 92, no. 25, Article ID 251103, 2008.

[64] M. Nagel, "Tapered Sommerfeld wire terahertz near-field imaging," Applied Physics Letters, vol. 94, no. 5, Article ID 051107, 2009.

[65] A. J. Adam, N. C. van der Valk, and P. C. Planken, "Measurement and calculation of the near field of a terahertz apertureless scanning optical microscope," Journal of the Optical Society of America B, vol. 24, no. 5, pp. 1080-1090, 2007.

[66] N. Abbasirad, D. Arslan, S. Fasold, I. Staude, F. Setzpfandt, and T. Pertsch, "Dual-probe SNOM for the near-field study of nanostructures," in Proceedings of the 2018 Photonics North (PN), Montreal, Canada, 2018.

[67] A. J. L. Adam, "Review of near-field terahertz measurement methods and their applications," Journal of Infrared, Millimeter, and Terahertz Waves, vol. 32, no. 8-9, pp. 976-1019, 2011.

[68] Y. Inouye and S. Kawata, "Near-field scanning optical microscope with a metallic probe tip," Optics Letters, vol. 19, no. 3, p. 159, 1994.

[69] A. Wokaun, J. P. Gordon, and P. F. Liao, "Radiation damping in surface-enhanced Raman scattering," Physical Review Letters, vol. 48, no. 14, pp. 957-960, 1982.

[70] S. C. Schneider, S. Grafström, and L. M. Eng, "Scattering nearfield optical microscopy of optically anisotropic systems," Physical Review B Condensed Matter, vol. 71, no. 11, p. 115418, 2005.

[71] I. S. Averbukh, B. M. Chernobrod, O. A. Sedletsky, and Y. Prior, "Coherent near field optical microscopy," Optics Communications, vol. 174, no. 1-4, pp. 33-41, 2000.

[72] F. Zenhausern, Y. Martin, and H. K. Wickramasinghe, "Scanning interferometric apertureless microscopy: optical imaging at 10 angstrom resolution," Science, vol. 269, no. 5227, pp. 1083-1085, 1995.

[73] P. Dean, "Apertureless near-field terahertz imaging using the self-mixing effect in a quantum cascade laser," Applied Physics Letters, vol. 108, no. 9, Article ID 091113, 2016. 